\title{
Oxyporus-Studien-II. Oxyporus (Oxyporus) procerus KraATz und 0. (Oxyporus) ruzickai spec. nov. aus China (Coleoptera, Staphylinidae, Oxyporinae) | Oxyporus procerus KraATz und O. ruzickai spec. nov.
}

Mit 16 Figuren und 1 Karte

MICHAEL SChÜLKE ${ }^{1}$

${ }^{1}$ Museum für Naturkunde Berlin, Invalidenstraße 43, 10115 Berlin, Germany. - mschuelke.berlin@t-online.de Published on 2017-12-08 DOI: $110.21248 /$ contrib.entomol. $67.2 .355-361_{1}$

\section{Zusammenfassung}

Typen und weiteres Material von Oxyporus (Oxyporus) procerus KraAtz, 1879 werden revidiert. Oxyporus chenpengi LI, 1992 wird mit O. procerus synonymisiert. Oxyporus (Oxyporus) ruzickai spec. nov. aus den chinesischen Provinzen Gansu und Yunnan wird beschrieben. Die behandelten Taxa werden abgebildet, ihre Verbreitung wird anhand einer Karte illustriert.

\section{Neue Taxa}

Oxyporus (Oxyporus) ruzickai spec. nov. - urn:lsid:zoobank.org:act:BD64C07C-1AEC-4800-9EC8-A7EFA28367CB

Key words

Coleoptera, Staphylinidae, Oxyporinae, Oxyporus, Palaearctic region, Russia, China, new species, new synonymy

\section{Abstract}

Types and additional material of Oxyporus (Oxyporus) procerus KRAATZ, 1879 are revised. Oxyporus chenpengi LI, 1992 is synonymised with O. procerus. Oxyporus (Oxyporus) ruzickai spec. nov. from the Chinese provinces Gansu and Yunnan is described. Both species are illustrated and their distributions are mapped.

\section{Einleitung}

In den letzten Jahrzehnten wurden vor allem aus China zahlreiche neue Arten der Gattung Oxyporus beschrieben. China ist ohne Zweifel das Land mit den zahlreichsten Vertretern der Unterfamilie Oxyporinae, bisher wurden (inklusive Taiwan) 52 Arten aus dem Gebiet beschrieben oder gemeldet (Herman 2001, SChülke \& SMetana 2014, Schülke 2016). Damit scheint die Diversität aber lange noch nicht erschöpft, zahlreiche bisher nicht bekannte Taxa liegen dem Autor zur Beschreibung vor. Leider wird die Bearbeitung durch den Umstand erschwert, das bisherige Beschreibungen sich unter anderem überwiegend auf Färbungsmerkmale beschränken und über die Variabilität zahlreicher Arten keinerlei Informationen vorliegen (siehe SCHÜLKE 2016). 
Einige der aus China beschriebenen Taxa waren bisher auf Grund der in chinesischer Sprache abgefassten Beschreibungen, sowie fehlender Abbildungen kaum deutbar. $\mathrm{Zu}$ diesen Arten gehört Oxyporus chenpengi J.-K. LI, 1992, beschrieben aus der nordchinesischen Provinz Jilin. Die chinesische Originalbeschreibung enthält keinerlei Abbildungen der in der Originalbeschreibung O. chenpongi genannten Art. Bei der Schreibweise des Artnamens in der Originalbeschreibung handelt es sich offenbar um einen Lapsus, in einer der Beschreibung vorangehenden Artenliste wird sie richtig als O. chenpengi (Li's Kollegen P. Chen gewidmet) geführt. Der Fundort der Art in der chinesischen Provinz Jilin legte den Verdacht nahe, dass sie mit einer der zahlreichen aus dem Fernen Osten Russlands und Korea beschriebenen oder gemeldeten Arten identisch sein könnte. Alle Bemühungen Typenmaterial des Autors zum Studium zu erhalten erwiesen sich jedoch als ergebnislos. Eine neue Arbeit unter Beteiligung des Autors (Li, Zhang \& Zhang 2015), die eine Abbildung von $O$. chenpengi enthält, erlaubt jedoch jetzt eine Klärung der Identität dieser Art, die als Synonym zu O. procerus KRAATZ, 1879 eingezogen wird. Zugleich ergibt sich damit die Möglichkeit eine weitere O. procerus ähnliche Art zu beschreiben, was nachfolgend unter dem Namen Oxyporus ruzickai spec. nov. erfolgt.

\section{Material und Methoden}

Die in dieser Arbeit verwendete Methodik folgt früheren Arbeiten (SchüLKe 2016). Messwerte wurden mit Hilfe eines Okular-Mikrometers bei Vergrößerungen von 20x und 70x gewonnen. Aufnahmen im Durchlicht wurden mit einer Digitalkamera vom Typ Nikon Coolpix 990 und einem Digital-Adapter der Firma LM-Scope mit Hilfe eines Mikroskops der Firma PZO (Warschau) angefertigt, Habitus-Aufnahmen mit einer Kamera vom Typ Canon EOS 450D mit Makroobjektiv. Zur Nachbearbeitung der Aufnahmen und Montage der Tafeln wurden Micrografx Picture Publisher 6.0 und CombineZP verwendet.

Die gewonnenen Messwerte sind wie folgt abgekürzt: Augenlänge von oben gemessen (AL), Maximale Kopfbreite $(\mathrm{KBr})$, Kopflänge (Vorderrand des Clypeus bis zur Halsabschnürung) (KL), Maximale Breite des Pronotums (HBr), Länge des Pronotums (Mittellinie) (HL), Maximale Elytrenbreite (zusammen) ( $\mathrm{FBr}$ ), Nahtlänge der Elytren (FNL), Schulterlänge der Elytren(FSL), Schläfenlänge (Augenhinterrand bis Halsabschnürung) (SL); Vorderkörperlänge (Vorderrand des Clypeus bis zum Hinterrand der Elytren) (VKL), Gesamtlänge bei herauspräparierter Hinterleibsspitze (GL), Länge des Aedoeagus-Medianlobus (AedL).

Das in diesem Beitrag untersuchte Material befindet sich in folgenden Sammlungen:

MNB Museum für Naturkunde Berlin (J. Frisch, J. Willers, B. Jaeger)
NHMW Naturhistorisches Museum Wien (H. Schillhammer)

NMP National Museum Prag (J. Hájek, M. Fikáček)

SDEI Senckenberg Deutsches Entomologisches Institut (L. Behne)

cPüt Sammlung A. Pütz (Eisenhüttenstadt)

cSch Sammlung M. Schülke (Museum für Naturkunde Berlin)

cSha Sammlung A. Shavrin (Daugavpils).

Oxyporus (Oxyporus) procerus KRAATZ, 1879

(= O. chenpengi Lı, 1992, syn. nov.)

Fig. 1-2, 5-10, Karte 1

Oxyporus procerus KraATZ, 1879: 122 (Locus typicus: Amur) Oxyporus procerus KraAtz, 1879: Fauvel 1879: 76; Heyden 1881: 79; Bernhauer \& SCHUbERT 1911: 149; SCHEERPELTZ 1925: 348; Alexandrov 1933: 1; Kirschenblat 1938: 539; Kryzhanovsky et.al. 1973: 148; Shavrin \& Berlov 1999: 10; Herman 2001: 1987; Smetana 2004: 536; SHABALIN 2012: 414; SCHÜLKE \& SMETANA 2015: 796.

Oxyporus chenpengi LI, 1992: 57 (Locus typicus: China; Jilin province, Liangdaobaihe, Changbaishan, $2000 \mathrm{~m}$ ) [teilweise irrtümlich als $O$. chenpongi].

Oxyporus chenpengi LI, 1992: LI 1993: 24; Herman 2001: 1977; Smetana 2004: 536; Schülke \& SMetana 2015: 796; LI, ZHANG \& ZHANG 2015: 60.

Untersuchtes Typenmaterial (procerus): „Amur Christoph 77 / Holotypus [rot] / coll. Kraatz / Oxyporus procerus KR. / coll. DEI Eberswalde / procerus Amur bor. / Oxyporus procerus Kraatz det. M.-H. Kim 2005“, $1 \mathrm{Ex}(\mathrm{SDEI})$.

Die genaue Herkunft des Holotypus lässt sich leider auch anhand der von Christoph publizierten Reisebeschreibungen (CHRIstoph 1878, 1880) nicht feststellen, jedoch eingrenzen. Christoph sammelte im Jahre 1876 bei Radde [Rajon Oblutschenski im Jüdischen Gebiet] am Amur und reiste am 20. August 1876 von dort über Chabarovsk nach Nikolsk, dem heutigen Ussurijsk, wo er den Winter 1876/1877 verbrachte. Im Frühjahr 1877 reiste er von dort weiter nach Wladiwostok, in dessen Umgebung er sich bis zu seiner Abreise im September 1877 aufhielt. Der Holotypus von O. procerus stammt also wahrscheinlich aus der Umgebung von Ussurijsk oder Wladiwostok, wo die Art auch heute noch regelmäßig gefunden wird, nicht vom Amur, wie in der Originalbeschreibung angegeben.

Weiteres untersuchtes Material: Russland: Region Primorje: Rajon Ussurijsk, Umgebung Kamenuschka, Ussuri Schutzgebiet, 21.-23.[? Monat unleserlich].1990, leg. A. Schwarz, 1 Ex. (MNB); 20 km N Artjom, 200-350 m, Kamenuschka, 15.V.-6.VI.2002, leg. A. Plutenko, 20 Ex. (MNB, cSch); N Artjom, Flusstal der Kamenuschka, 100-350 m, 15.-30.V.2005, leg. A. Plutenko, 21 Ex. 

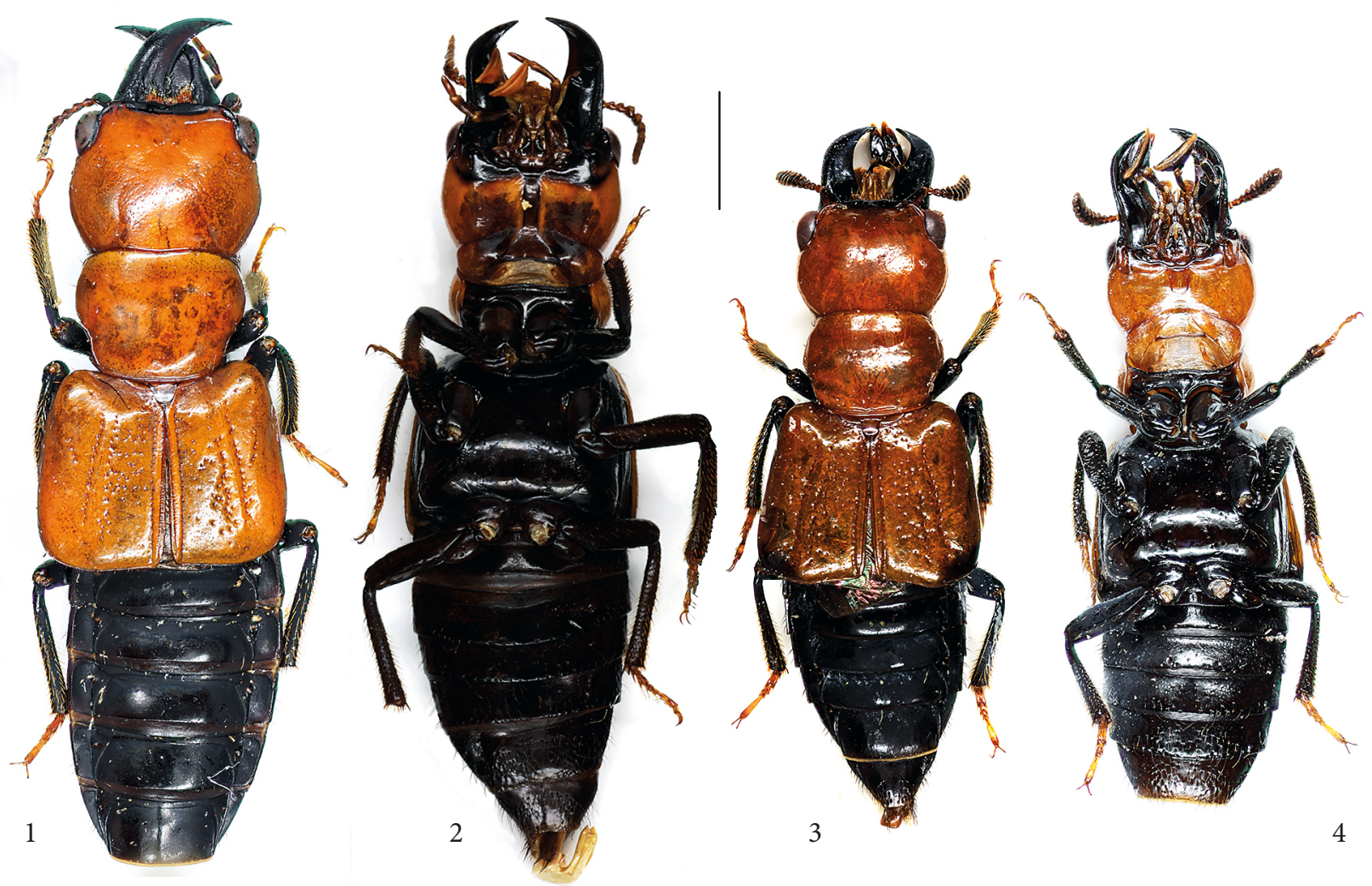

4

Fig. 1-4: Habitus von Oxyporus (Oxyporus) procerus KraAtz, Russland, Primorje, Umg. Artjom, dorsal (1), ventral (2) und O. (O.) ruzickai spec. nov., Holotypus, dorsal (3), ventral (4). Maßstab 2 mm.

(cSch); Ussuri Schutzgebiet, Kordon Peishula, 13.-20. VIII.1998, leg. J. Sundukov, 4 Ex. (cSch); Ussuri Schutzgebiet, Kamenuschka, 21.VII.1990, leg. A. Pütz, 2 Ex. (SDEI); Kreis Arsenjew: Umgebung Arsenjew, VI.1994, leg. Štrba, 1 Ex. (MNB); Umgebung Arsenjew, 27.V.-5. VII.1991, leg. O. Sausa, 5 Ex. (NHMW, cSch); Umgebung Arsenjew, VI.1991, leg. Štrba, 1 Ex. (NHMW); Kreis Artjom: S Artjom, Flusstal der Ozernyi kluytch, 100-300 m, 10.V.-5.VI.2002, leg. Plutenko, 1 Ex. (cSch); Artyom, $35 \mathrm{~km}$ NE Wladiwostok, Flusstal der Ozernyi kluytch, 10.V.-7.VI.2005, leg. A. Plutenko, 13 Ex. (cSch); Kreis Partisansk: Ussuri, Sutschan, 2 Ex. (NMP); Rajon Laso: Elomovsky Flusstal, 12.VII.1995, leg. Sundukov, 3 Ex. (cPüt, cSch); Laso Nationalpark, Flusstal des Sobolnij, 400-800 m, 27.-28.VI.1994, leg. Sundukov, 2 Ex. (cPüt, cSch); Rajon Laso, Laso Nationalpark, Unterlauf des Flusses Priamuschka, 8.-11.VII.2007, leg. J. Sundukov, 5 Ex. (cSha, cSch); Region Chabarovsk: Rajon Bikin: Birskoe, 16.VI.1958, 1 Ex. (NHMW).

China: Hebei: Chengde, Wulingshan, $1178 \mathrm{~m}$, Qingliang area, $40^{\circ} 36^{\prime} 46^{\prime \prime} \mathrm{N}, 117^{\circ} 27^{\prime} 52^{\prime \prime E}$, 11.VIII.2016, leg. P. Kment, 1 ㅇ (NMP).

Die in chinesisch ohne Abbildungen oder englische Zusammenfassung verfasste Originalbeschreibung von Oxyporus chenpengi LI 1992 war bisher nicht deutbar. Typenmaterial der aus der chinesischen Provinz Jilin beschriebenen Art war nicht zur Untersuchung zugänglich. Eine kürzlich erfolgte weitere Publikation des Autors ermöglicht aber eine Deutung der Art. In einer Arbeit mit dem Titel "Yuanse zhongguo dongbei turang jiachong tujian (yinchichong lei, nibujia lei)" [Primary color illustration of soil beetles in Northeast China] veröffentlichen LI, ZHANG \& ZHANG (2015) eine Abbildung der Art (Seite 60: Abb. 105), die ihre Identität mit Oxyporus procerus KraAtz belegt. Als einzigen Unterschied zu $O$. procerus geben die Autoren an, daß O. chenpengi weniger Punkte auf den Elytren besitzen soll [Übersetzung Z.-W. Yin], ein Merkmal, das einer bedeutenden Variabilität unterliegt und zur Artunterscheidung nicht geeignet ist. Die Art besitzt unter den im Gebiet vorkommenden Gattungsvertretern ein einmaliges Färbungsmuster und ist in der benachbarten russischen Region Primorje weit verbreitet und nicht selten. Aus der heutigen chinesischen Provinz Heilongjiang wurde die Art bereits durch Alexandrov (1933) gemeldet. Das aus der Provinz Hebei vorliegende weibliche Exemplar unterscheidet sich außer durch die vollständig dunkle Färbung der Unterseite des Kopfes nicht von Exemplaren aus dem Fernen Osten Russlands. Die Färbung der Kopfunterseite variiert aber auch bei russischen Exemplaren von hell über unscharf verdunkelt bis zu Exemplaren mit deutlich abgesetzten dunklen Bereichen, spezifische Färbungsunterschiede lassen sich nicht feststellen.

Verbreitung: Bisher aus den russischen Regionen Primorje (inkl. Sachalin (Shavrin \& Berlov 1999)) und Chabarovsk (Shabalin 2012), sowie aus den chinesischen Provinzen Hebei, Heilongjiang und Jilin bekannt (Karte 1). 

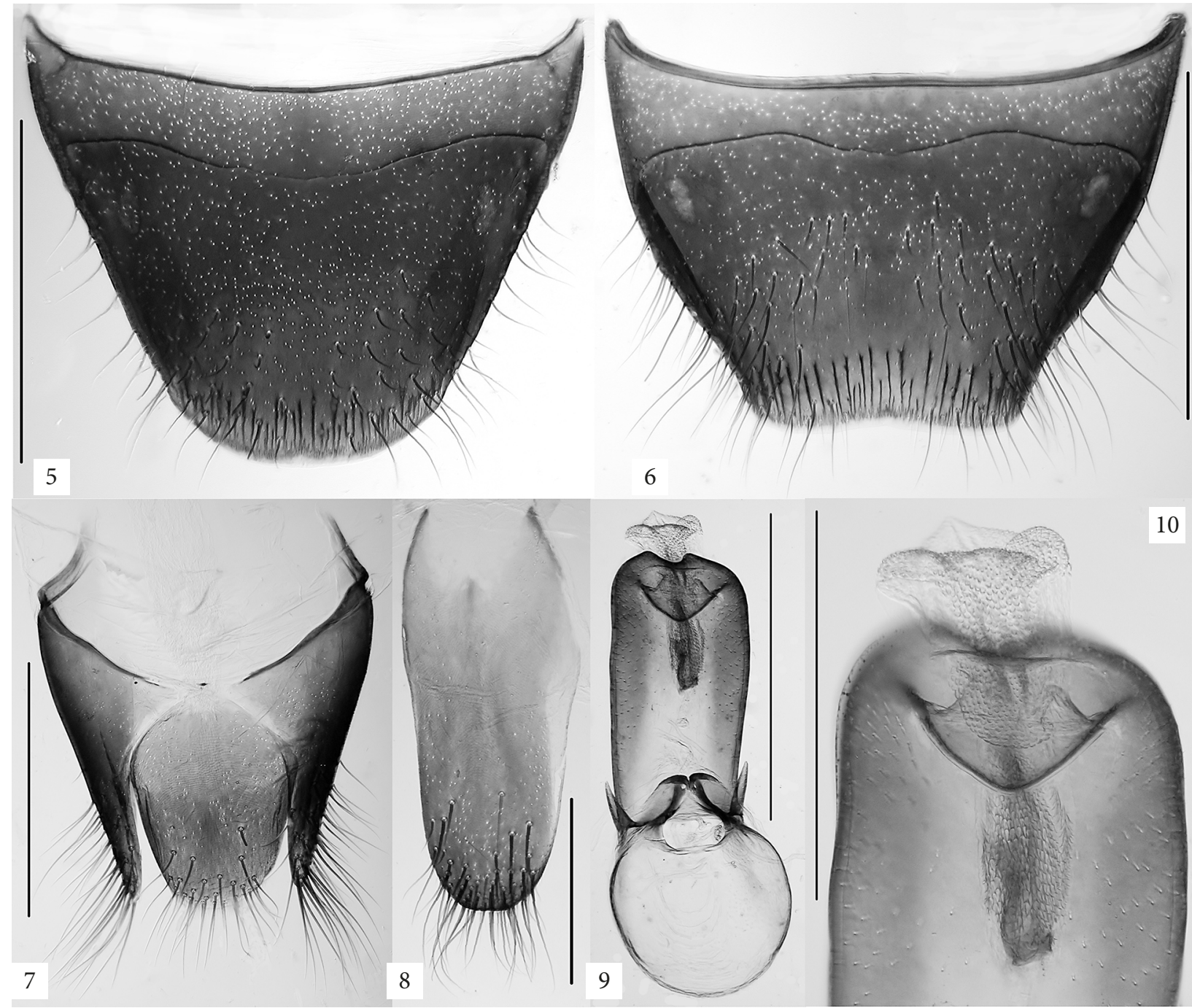

Fig. 5-10: Oxyporus (Oxyporus) procerus KraAtz, Russland, Primorje, Umg. Artjom: Tergit VIII (5), Sternit VIII (6), Tergite IX-X (7), Sternit IX (8), Aedoeagus (ventral) (9), Aedoeagus, Spitze des Medianlobus mit teilweise ausgestülpten Innenstrukturen (10). Maßstäbe $1 \mathrm{~mm}(5-7,9), 0,5 \mathrm{~mm}(8,10)$.

\section{Oxyporus (Oxyporus) ruzickai spec. nov.

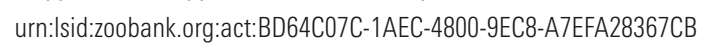 Fig. 3-4, 11-16, Karte 1}

Holotypus- $o^{\star}:$ „CHINA: Gansu province, LAZIKOU valley, $2150 \mathrm{~m}, 34^{\circ} 08.0^{\prime} \mathrm{N}, 103^{\circ} 54.5^{\prime} \mathrm{E}$ (GPS), 27.VI.2005, J. Hájek, D. Král \& J. Růžička leg. [Ch 7] / individually in excrements and on vegetation near the stream, flowering bushes, on palisade near village / HOLOTYPUS- ${ }^{\star}$ Oxyporus ruzickai spec. nov. det. M. Schülke 2016 [rot]“, $1 \sigma^{\star}(\mathrm{cSch})$.

Paratypus- $0^{\star}$ : „Cina Yunnan 1.-19.VII. HEISHUI $35 \mathrm{~km}$ N Lijiang $27^{\circ} 13^{\prime} \mathrm{N}, 100^{\circ} 19^{\prime}$ E E. Jendek leg. 1992 / PARATYPUS- $\sigma^{\star}$ Oxyporus ruzickai spec. nov. det. M. Schülke 2016 [gelb]“, 1 ơ (NHMW).

Beschreibung: Habitus wie in Fig. 3-4. Färbung sehr ähnlich O. procerus KraATZ 1879 (Fig. 1-2), aus dem Fernen Osten Russlands: Kopf bis auf den schmalen Vorderrand des Clypeus, Pronotum, Scutellum und
Elytren gelb. Posterolaterale Winkel der Elytren mit kleiner und unauffälliger Verdunkelung. Abdomen, Tibien und Femora schwarz, Tarsen gelb. Mandibeln schwarz, Labrum gelb, Maxillarpalpen dunkelbraun bis schwarz mit etwas helleren Spitzen der Apikalglieder, Labialpalpen ebenfalls dunkelbraun bis schwarz, Endglied an der Vorderkante heller. Antennen dunkelbraun mit etwas helleren Gliedern II und III sowie aufgehellten Seiten der Glieder V-XI.

Messwerte (Holotypus): AL 0,73; SL 1,17; $\operatorname{KBr} 2,59$; KL 2,05; HBr 2,39; HL 1,78; FNL 2,39; FSL 3, 17; FBr 3,56; VKL 6,71; GL 10,43; AedL 1,18. Indizes: AL/SL 0,63; $\mathrm{KBr} / \mathrm{KL}$ 1,26; KBr/HBr 1,08; HBr/HL 1,34; HL/FNL 0,74; FSL/FBr 0,89.

Messwerte (Paratypus): AL 0,68; SL 1,11; $\operatorname{KBr} 2,37$; KL 1,95; HBr 2,15; HL 1,76; FNL 2,26; FSL 2,88; FBr 3,37; VKL 6,15; GL 11,86. Indizes: AL/SL 0,61; $\mathrm{KBr} / \mathrm{KL} 1,21 ; \mathrm{KBr} / \mathrm{HBr}$ 1,10; HBr/HL 1,22; HL/FNL 0,78; FSL/FBr 0,86 .

Längenverhältnisse der Antennenglieder I-XI: 30:10:11:1 0:10,5:10,5:11,5:11,5: 10,5:10:15. Verhältnis Länge/Breite 

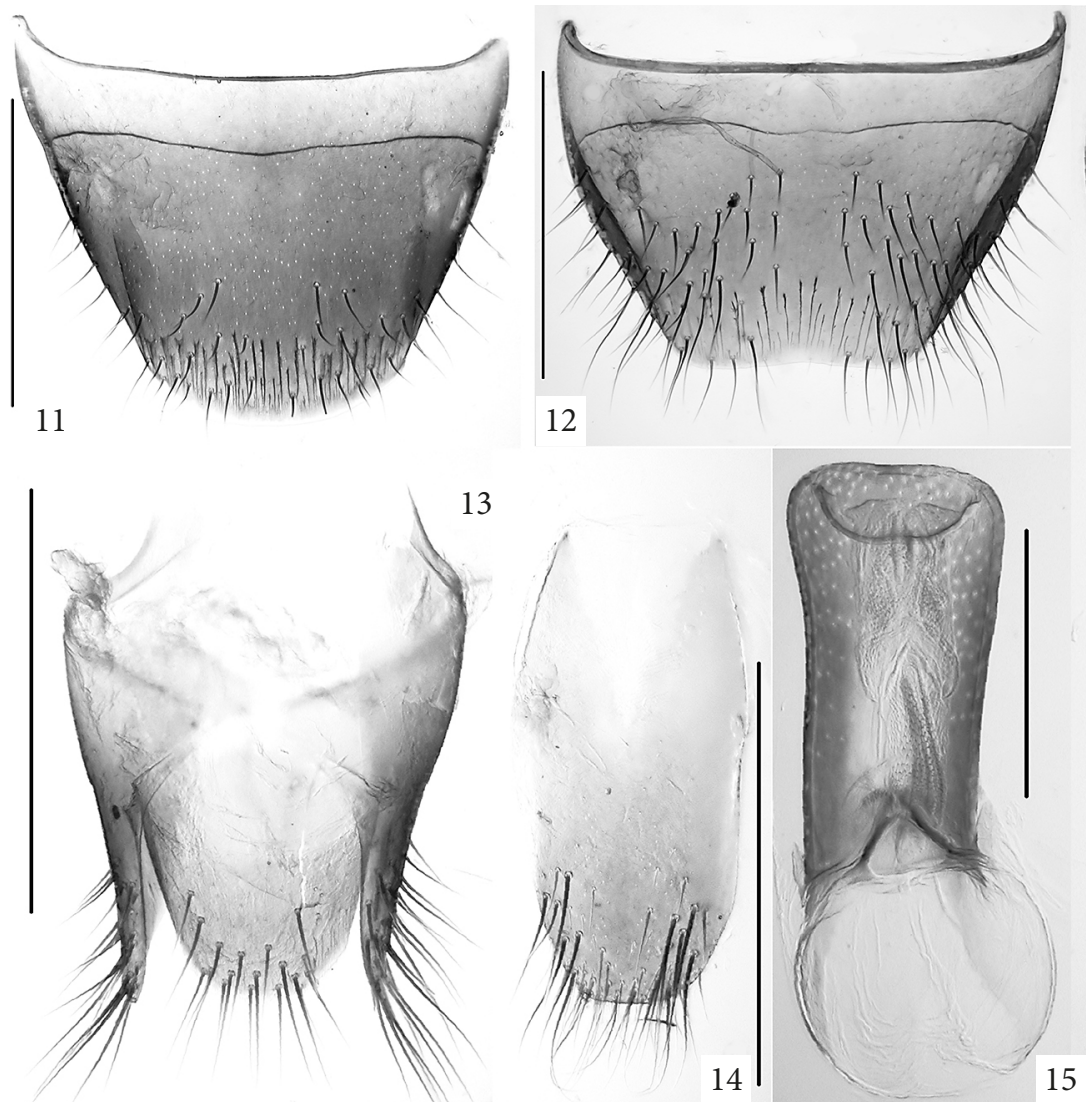

13
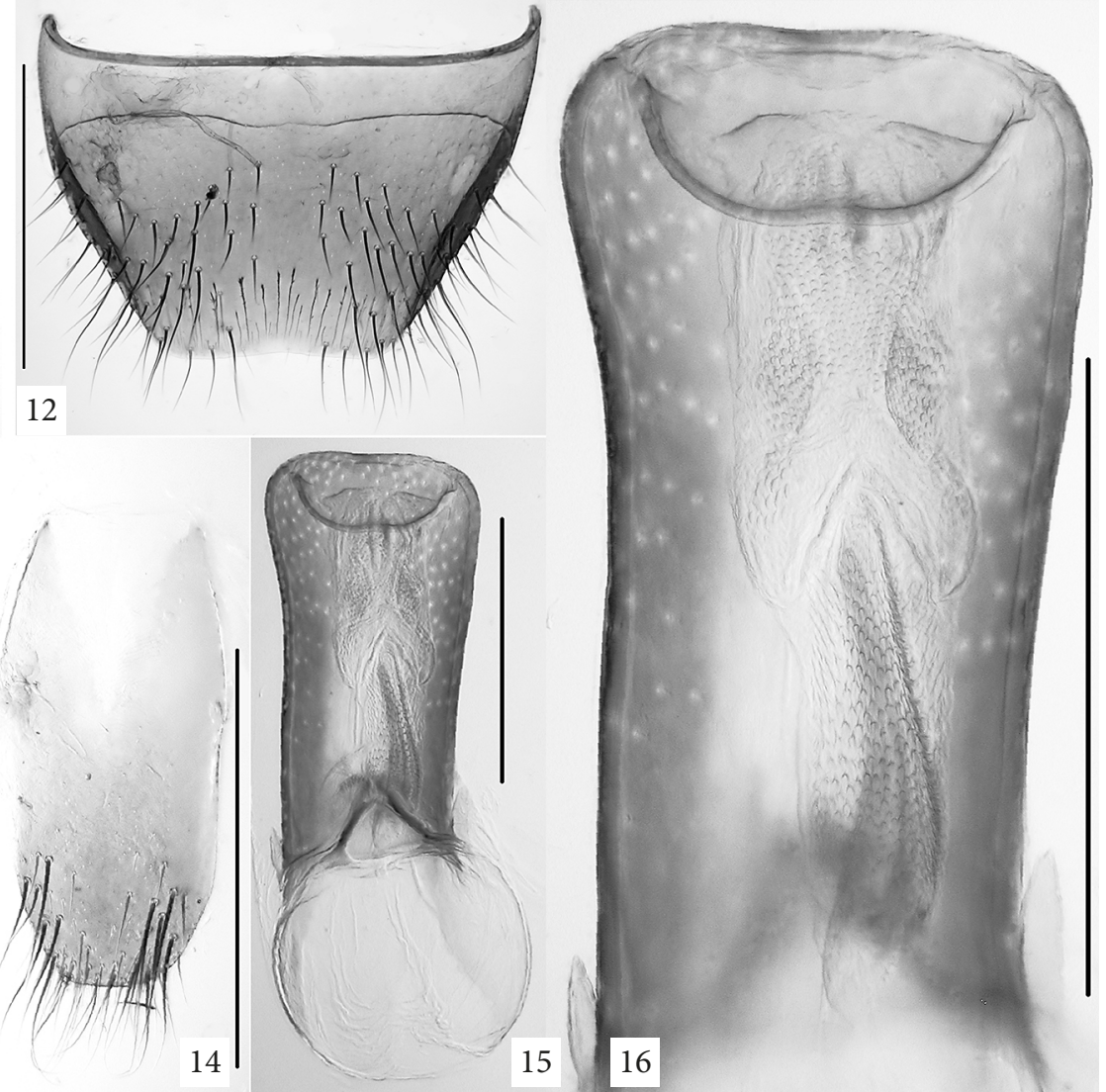

Fig. 11-16: Oxyporus (Oxyporus) ruzickai spec. nov., Holotypus (11-14), Paratypus (15-16): Tergit VIII (11), Sternit VIII (12), Tergite IX-X (13), Sternit IX (14), Aedoeagus (ventral) (15), Aedoeagus, Spitze des Medianlobus mit Innenstrukturen(16). Maßstäbe $1 \mathrm{~mm}$ (11-14), 0,5 mm (15-16).

von Glied I: 2,14; III 1,00; IV: 0,87; V: 0,68; VI: 0,55; VIII: 0,55; X: 0,50; XI 0,94.

Kopf bis zur Halsabschnürung quer (KBr/KL 1,211,26), seine breiteste Stelle befindet sich deutlich hinter den Augen. Vorderrand des Clypeus über den Einlenkungen der Antennen etwas wulstig aufgebogen, in der Mitte nach vorn erweitert, die Mitte des Vorderrandes nur wenig ausgerandet. Augen groß (AL/SL 0,61-0,63), Supraorbitalborsten beiderseits verdoppelt. Mandibeln kräftig und gestreckt, ihr Außenrand im basalen Teil wulstförmig erhaben. Linke Mandibel mit deutlichem Mittelzahn auf der Unterseite. Oberfläche des Kopfes mit feiner Mikropunktur, ohne deutlich sichtbare Mikroskulptur. Antennen kurz und abgeplattet, ab Glied V deutlich quer, nach Innen stärker als nach Außen erweitert, die erweiterten Seitenteile matt und fein tomentiert. Apex der Glieder I-X, Glied XI auch entlang der Seiten mit kräftigeren Tastborsten. Pronotum deutlich quer (HBr/HL 1,22-1,34), Querfurche im vorderen Drittel des Pronotums in der Mitte nur undeutlich. Randung des Pronotums vorn in der Mitte fehlend, erst vom Niveau der von innen gesehen dritten Vorderrandborste deutlich ausgebildet, Randung an den Seiten und hinten kräftig. Seiten des Pronotums in der hinteren Hälfte beiderseits mit zwei Eindrücken. Oberfläche des Pronotums sonst ohne Eindrücke, nur sehr unregelmäßig und weitläufig fein punktiert, ohne deutlich sichtbare Mikroskulptur. Elytren mit kräftiger Schulterbeule, deutlich breiter als
Kopf und Pronotum ( $\mathrm{HBr} / \mathrm{FBr} 0,64-0,67)$, zusammen etwas breiter als lang (FSL/FBr 0,86-0,89) und an der Naht deutlich länger als das Pronotum (HL/FNL 0,74-0,78). Oberfläche glänzend mit kräftiger, in einer Längsfurche stehender Humeralpunktreihe, einer nicht vertieft stehenden interioren Punktreihe sowie etwa 15-25 unregelmäßig zwischen interiorer Punktreihe und Naht verteilten Einzelpunkten. Suturalpunktreihe in feiner Längsfurche, aus viel kleineren Punkten bestehend. Seiten und Hinterrand der Elytren deutlich gerandet.

Innerhalb der Humeralpunktreihe befinden sich je drei borstentragende Punkte, weitere befinden sich längs des Außenrandes der Elytren und zwischen Suturalpunktreihe und Naht, fünf borstentragende Punkte sind um die Schulterbeule herum angeordnet. Oberfläche der Elytren ohne deutlich sichtbare Mikroskulptur.

Abdomen glänzend, die Tergite nur mit sehr undeutlicher und feiner Punktur, die erst auf Tergit VIII kräftiger wird. Tergite III und IV mit einem Paar Tomentflecken, Hinterrand von Tergit VII mit deutlichem Hautsaum (palisade fringe). Tergite mit sehr undeutlicher querwelliger Mikroskulptur, die erst auf Tergit VIII deutlicher ist. Beine wie bei anderen Arten gebildet.

Männchen: Sternite III-V nur vor dem Hinterrand und in der Mitte, Sternit VI in der Hinterhälfte, Sternit VII auf der ganzen Oberfläche kräftig punktiert und lang beborstet. Sternit VIII (Fig. 12) am Hinterrand annähernd gerade abgestutzt, in ganz ähnlicher Weise wie 


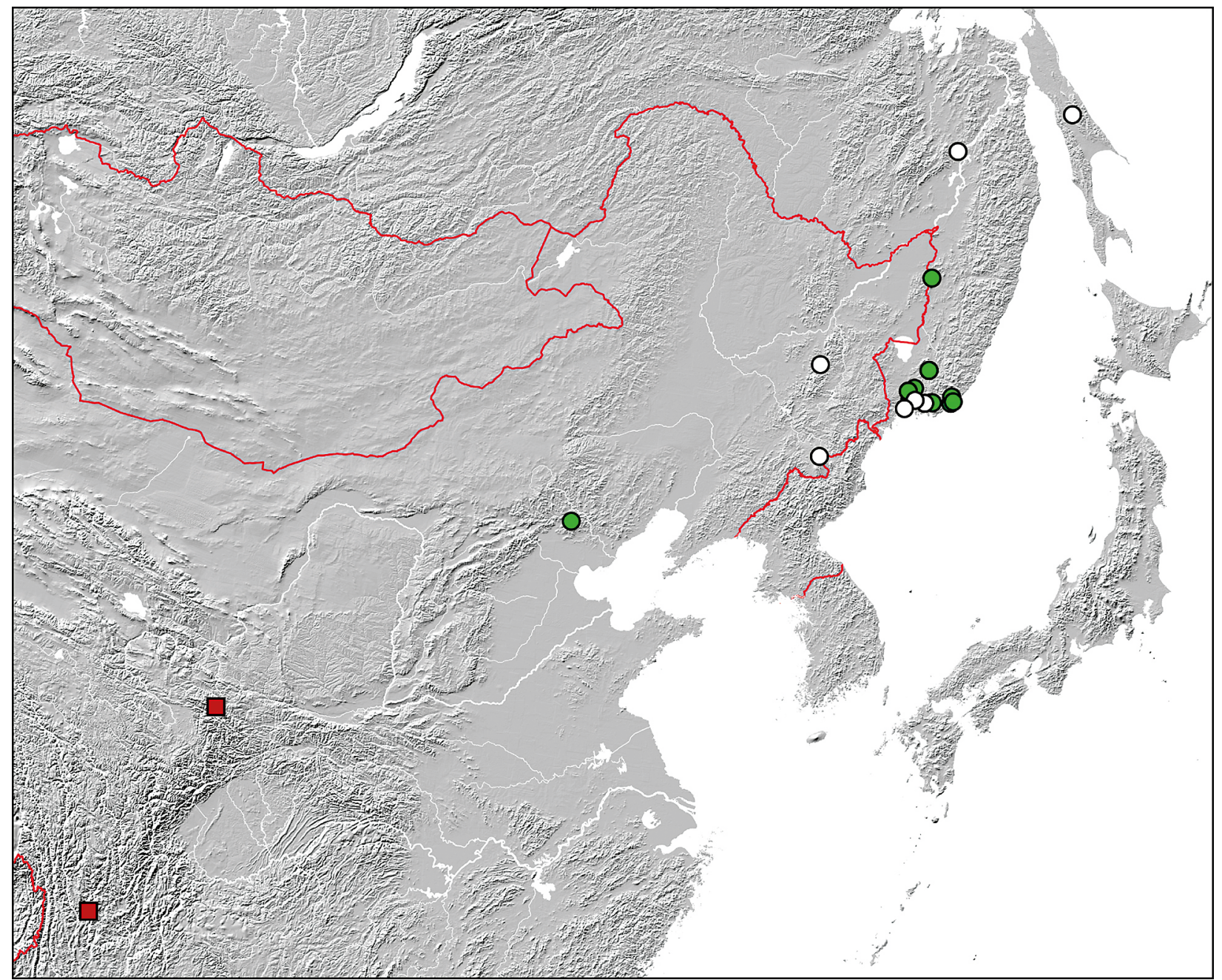

Karte 1: Verbreitung von Oxyporus (Oxyporus) procerus KraAtz (grüne Kreise - untersuchte Belege, weiße Kreise - glaubhafte Literaturangaben) und O. (O.) ruzickai spec. nov. (rote Quadrate).

bei O. procerus (Fig. 6) beborstet. Tergit VIII (Fig. 11) etwas deutlicher quer als bei O. procerus (Fig. 5), Beborstung in der apikalen Hälfte des Tergits etwas weniger umfangreich. Tergite IX und X (Fig. 13) ähnlich wie bei O. procerus (Fig. 7), Sternit IX (Fig. 14) etwas kürzer als bei O. procerus (siehe Fig. 8). Aedoeagus (Fig. 15-16) kleiner als bei O. procerus (Fig. 9-10), leicht asymmetrisch, zum Apex des Medianlobus etwas erweitert. Apex des Medianlobus ohne Einkerbung, Rand der apikalen Öffnung des Medianlobus breiter und weniger tief ausgeschnitten. Innenstrukturen (Fig. 16) unauffällig, aus borstentragenden, häutigen Strukturen bestehend.

Weibchen: unbekannt.

Differentialdiagnose: Oxyporus ruzickai spec. nov. ist O. procerus in Färbung und Habitus außerordentlich ähnlich, die beiden vorliegenden Exemplare sind etwas kleiner als durchschnittliche Exemplare von O. procerus, liegen aber noch in der Variationsbreite dieser Art. Die neue Art unterscheidet sich von $O$. procerus aber durch die folgenden Merkmale: Posterolaterale Winkel der Elytren mit kleiner dunkler Makel, Maxillar- und Labialpalpen dunkel (bei O. procerus sind die Maxillarpalpen bei allen untersuchten Exemplaren hell, die Maxillarpalpen meist hell, bei einem Teil der Tiere jedoch auch teilweise oder ganz angedunkelt), weniger deutliche Ausrandung in der Mitte des Vorderrandes des Clypeus, größere Augen (AL/SL 0,61-0,63, bei O. procerus 0,45-0,55) und verdoppelte Supraorbitalborsten, sowie den Bau des Aedoeagus, der bei O. procerus am Apex des Medianlobus deutlich eingekerbt ist.

Leider wurden einige Merkmale bisher bei der Beschreibung von Oxyporus-Arten kaum berücksichtigt. So ist mir nur zwei Beschreibungen bekannt, in denen von doppelter oder mehrfacher Supraorbitalbeborstung einer Oxyporus-Art berichtet wird (HAYASHI 1985, SCHÜLKE 2016). Wenn auch die überwiegende Mehrheit der Oxyporus-Arten nur einfache Supraorbitalborsten trägt, müssen alle beschriebenen Arten auf dieses Merkmal hin untersucht werden.

Verbreitung und Bionomie: Bisher nur von den Fundorten der Typen im Süden von Gansu und im Norden von Yunnan bekannt (Karte 1). Die auf den Etiketten des Holotypus angegebenen Fundumstände lassen sich der Art nicht zuordnen, zweifellos ist sie wie andere Gattungsvertreter mycetophag. Die beiden, weit auseinander liegenden Fundorte lassen vermuten, dass die 
Art zumindest im westlichen China entlang der Ostabdachung des tibetischen Hochlandes weit verbreitet ist.

Derivatio nominis: Die neue Art wird dem Leiodidenund Silphidenspezialisten Jan Růžička (Prag), dem ich auch für die Überlassung des Holotypus der Art zu danken habe, in freundschaftlicher Anerkennung gewidmet.

\section{Danksagung}

Herzlicher Dank gilt allen im Abschnitt Material und Methoden genannten Kollegen und Institutionen für die Möglichkeit ihr Material zu bearbeiten. Zi-Wei Yin (Shanghai) danke ich für die Übersetzung von Passagen aus LI, Zhang \& Zhang (2015), V. Assing (Hannover) für die Überprüfung der englischsprachigen Zusammenfassung.

\section{Literaturverzeichnis}

Alexandrov, A. J. 1933: Some information about the little-known species - Oxyporus procerus KR. - Entomologisches Nachrichtenblatt 7(1): 1-2.

Bernhauer, M. \& Schubert, K. 1911: Staphylinidae II.: 87-190. - In: Schenkling, S. (ed): Coleopterorum Catalogus auspiciis et auxilio. pars 29 - W. Junk, Berlin.

Christoph, H. 1878: Nach und vom Amur. - Entomologische Zeitung Stettin 39: 201-219, 401-410. - https:// www.biodiversitylibrary.org/page/8988399\#page/205/, mode/1up, - [accessed 2017/11/15].

Christoph, H. 1880: Neue Lepidopteren des Amurgebietes. - Bulletin de la Société Impériale des Naturalistes de Moscou 55(3): 20-121.

FAuvel, A. 1879: Annuaire entomologique pour 1879 (7e année). - Fauvel \& Bouquet, Caen \& Paris: $135+$ 1 pp.

Hayashi, Y. 1985: Notes on Staphylinidae (Col.) from Taiwan, IV. - Entomological Review of Japan 40(2): 81-84.

Herman, L. H. 2001: Catalogue of the Staphylinidae (Insecta: Coleoptera). 1758 to the end of the millenium. - Bulletin of the American Museum of Natural History 265(1-7): vi + 4218 p.

Heyden, L. von 1881: Catalog der Coleopteren von Sibirien mit Einschluss derjenigen der Turanischen Länder, Turkestans und der chinesischen Grenzgebiete. - Deutsche Entomologische Zeitschrift 24 [1880] (Sonderheft): 1-96.

Kirschenblatt, J. D. 1938: O nekotorykh dalnevostochnykh shukakh-stafilinidakh [On some far eastern staphylinid beetles]. - Trudy gidrobiologitsheskoi expedizii SIN AN 1934 g. na Japonskoe More 1: 527-536. (russisch mit englischer Zusammenfassung).
KraAtz, G. 1879: Neue Käfer vom Amur. - Deutsche Entomologische Zeitschrift 23: 121-144. - https:// Www.biodiversitylibrary.org/item/97573\#page/ $1277_{1}$ mode/1up, [accessed $2017 / \overline{1} 1 / \overline{1} \overline{4}]$.

KrYzhanovsky, O. L.; Tikhomirova, A. L. \& FilaTOVA, L. D. 1973: Stafilinidy (Coleoptera, Staphylinidae) yuzhnogo Primorya [Staphylinid beetles (Coleoptera, Staphylinidae) of South Primorye), S. 144-173. - In: Gilyarov, M. [Red.]: Ekologiya pochvennykh bespozvonochnykh. Nauka, Moskau: 226 S. (russisch).

LI, J.-K. 1992: [The Coleoptera fauna of Northeast China.]. - Jilin Education Publishing House, Jilin: 205 S. (chinesisch, teilweise englisch).

LI, J.-K. 1993: [The rove beetles of Northeast China.] S. 1-63, 151-163. - In: LI, J.-K. \& Chen, P.: Studies on fauna and ecogeography of soil animal. - Northeast Normal University Press, Changchun: 265 S. (chinesisch).

LI, J.-K.; Zhang, L.-M. \& Zhang, X.-P. 2015: [Primary color illustration of soil beetles in Northeast China. Staphylinidae and Tenebrionoidea.]. - Harbin Map Press, Harbin: 199 S. (chinesisch).

Scheerpeltz, O. 1925: Staphylinidae. - In: Winkler, A. [Hrsg.]: Catalogus Coleopterorum regionis palaearcticae. Pars 3 _ _ _ A. Winkler, _ Wien: Spalten 241-368. _ _ https://www.zobodat.at/pdf/MON-E'COL_0042_0001-1698.p $\overline{\mathrm{d}}$ [accessed $2017 / \overline{1} / 1 \overline{4}]$.

SCHÜLKE, M. 2016: Oxyporus hiekei n. sp. aus China, eine Art mit außergewöhnlichem Sexualdimorphismus (Coleoptera, Staphylinidae, Oxyporinae). - Entomologische Blätter und Coleoptera 112(1): 365-372.

Schülke, M. \& Smetana, A. 2015: Staphylinidae, S. 304-1134. - In: LöBL, I. \& LöвL, D. (eds): Hydrophiloidea - Staphylinoidea. Volume 2, Revised and updated edition: Catalogue of Palaeactic Coleoptera. - Brill, Leiden und Boston: I-XXV, 1-1702. - ISBN 9789004289925.

Shabalin, S. A. 2012: A review of the rove beetle subfamily Oxyporinae (Coleoptera, Staphylinidae) of the Russian Far East. - Euroasian Entomological Journal 11(5): 412-416. (russisch mit englischer Zusammenfassung).

Shavrin, A. V. \& Berlov, E. Ya. 1999: K faune shukov stafilinid (Coleoptera, Staphylinidae) ostrova Sakhalin [To the fauna of the rove beetles (Coleoptera, Staphylinidae) of Sakhalin Island]. - Vestnik of the Irkutsk State Agricultural Academy 18: 9-12. (russisch).

Smetana, A. 2004: Staphylinidae, S. 237-698. - In: Löbl, I. \& Smetana, A. (Hrsg.): Catalogue of Palaeactic Coleoptera. Volume 2. Hydrophiloidea - Histeroidea - Staphylinoidea. - Apollo Book, Stenstrup: 942 S. - ISBN 8788757749. 


\section{Besprechung / Review}

Gaedike, R.; Nuss, M.; Steiner, A. \& Trusch, R. (Hrsg.) 2017: „Verzeichnis der Schmetterlinge Deutschlands (Lepidoptera)“. - Entomologische Nachrichten und Berichte, Beiheft 21, Dresden: 362 S. - ISSN 0232-5535.

Zuletzt wurde eine gesamte Checkliste für die Schmetterlinge Deutschlands im Jahr 1999 von Gaedike und Heinicke publiziert. Seitdem wurden viele neue Erkenntnisse gesammelt, weshalb hohe Anerkennung für die Herausgeber ausgesprochen werden muss, dass sie sich den Mühen unterzogen haben, nun eine Überarbeitung zu präsentieren. Diese ist rundherum gelungen.

Das Herzstück dieses Buchs sind natürlich die großen Tabellen, in denen alle für Deutschland bekannten 3682 Schmetterlingsarten aufgeführt sind. Diese Tabellen geben nicht nur den Nachweis für Deutschland als Ganzes an, sondern sind aufgeschlüsselt nach den Bundesländern. Hierbei wurden die Stadtstaaten Hamburg, Bremen und Berlin sinnvoller Weise den jeweiligen Flächenländern Schleswig-Holstein, Niedersachen und Brandenburg zugeschlagen. Besonders hervorgehoben werden muss auch, dass es sich nicht um eine reine Präsenz-AbsenzListe handelt, sondern dass die letzten Nachweise in vier
Zeitfenster aufgeschlüsselt sind, aktuell (also mit Nachweisen von 2001 bis 2016), von 1981 bis 2000, von 1901 bis 1980 und nur bis zum Jahr 1900. An diesen Tabellenteil schließt sich ein Kommentarteil an, der fast 200 Seiten umfasst und in dem eine unglaubliche Fülle an wichtigen Zusatzinformationen $\mathrm{zu}$ vielen der Arten zusammengetragen wurde. Im Tabellenteil ist jeweils vermerkt, wenn ein solcher Kommentar existiert und auf welcher Seite er sich befindet. Jeder Schmetterlingsfreund wird hier für sich persönlich interessante weitere Informationen finden. Im anschließenden Bildteil werden die Schmetterlingsfamilien mit jeweils typischen Vertretern vorgestellt. Abgerundet wird dieses epochale Werk durch ein umfängliches Literaturverzeichnis, in dem wichtige regionale Faunen und neuere Rote Listen nochmals extra ausgewiesen sind.

Insgesamt ist dieses aktualisierte Verzeichnis der Schmetterlinge Deutschlands ein bedeutendes Werk und ein absolutes Must-Have für jeden an Schmetterlingen wirklich Interessierten.

Th. SchmitT 